\title{
Functional assessment of coronary artery stenosis by Doppler derived absolute and relative coronary blood flow velocity reserve in comparison with ${ }^{99 \mathrm{~m}}$ Tc MIBI SPECT
}

H J Verberne, J J Piek, R A M van Liebergen, K T Koch, J M Schroeder-Tanka, $\mathrm{E}$ A van Royen

\begin{abstract}
Objective-To determine the relation between the relative and absolute coronary blood flow velocity reserve (CFVR) compared with the results of ${ }^{99 \mathrm{~m}}$ Tc MIBI single photon emission computed tomography (SPECT).
\end{abstract}

Methods-In 37 patients with one vessel disease, ${ }^{99 \mathrm{~m}}$ Tc MIBI SPECT was performed before angioplasty, two to three weeks after angioplasty, and at six months' follow up. CFVR was measured distal to the stenosis (dCFVR) as well as in a reference coronary artery before angioplasty, immediately after angioplasty, and at late follow up. Relative CFVR (rCFVR) was calculated as the ratio between $\mathrm{dCFVR}$ and CFVR measured in the reference coronary artery. The optimal thresholds for reversible perfusion defects were calculated using receiver operating characteristic curves.

Results-The agreement for the full range of coronary artery stenosis $(n=107$, mean (SD) diameter stenosis $48(28) \%$, range 0-98\%) between dCFVR (cut off value 1.9) and rCFVR (cut off value 0.65 ) with ${ }^{99 \mathrm{~m}} \mathrm{Tc}$ MIBI SPECT was $81 \%$ and $85 \%$, respectively. In intermediate lesions ( $n=49$, diameter stenosis range $30-75 \%$ ) the agreement between dCFVR (cut off value 2.0 ) and ${ }^{99 \mathrm{~m}}$ Tc MIBI SPECT was $72 \%$, which increased to $78 \%$ using the rCFVR (cut off value 0.65 ). There was a strong linear relation between $\mathrm{dCFVR}$ and rCFVR $(r=0.93, \mathrm{p}<0.0001)$.

Conclusions-A best cut off value for dCFVR of 1.9 corresponds with a best cut off value of 0.65 for rCFVR, within the full range of coronary narrowings. Intracoronary blood flow velocity analysis could obviate the need for additional myocardial perfusion scintigraphy in the majority of patients.

(Heart 1999;82:509-514)

Keywords: intracoronary Doppler; relative coronary blood flow velocity reserve; ${ }^{99 \mathrm{~m}}$ Tc MIBI single photon emission computed tomography

Decisions regarding intracoronary interventions should be based on objective evidence of functional lesion severity. Coronary angiography still serves as a gold standard for the evaluation of coronary lesion severity. However, there are discrepancies between angiographic and functional estimates of coronary lesion severity. ${ }^{1-4}$ Unfortunately, numerous coronary interventions are performed without objective evidence of myocardial ischemia. ${ }^{5}$ Consequently, this may lead to inappropriate decisions regarding intracoronary revascularisation.

Experimental data have shown that coronary flow velocity reserve (CFVR), the ratio of maximal to resting blood flow, is a sensitive and reliable index of the functional severity of a coronary stenosis. ${ }^{6}$ The development of a Doppler tipped angioplasty guide wire has made it possible to measure flow velocity distal to a coronary lesion in the clinical setting. ${ }^{7}$ Recent studies indicate a good agreement between the distal CFVR (dCFVR), assessed with the Doppler guide wire, and the results of perfusion scintigraphy. ${ }^{8-11}$ However, the absolute CFVR is limited by its dependency on haemodynamic variables. ${ }^{12-14}$ The relative CFVR (rCFVR), the ratio of poststenotic coronary flow velocity to the coronary flow velocity in a normal reference vessel, is compared to the absolute coronary flow velocity relatively independent of these variables. ${ }^{6}$

We aimed to evaluate the relation between rCFVR and absolute CFVR and the results of myocardial perfusion scintigraphy within the full range of coronary lesion severity. In order to obtain these data we assessed both dCFVR, rCFVR, and ${ }^{99 \mathrm{~m}}$ Tc MIBI single photon emission computed tomography (SPECT) in patients before angioplasty, immediately after angioplasty, and at six months' follow up.

\section{Methods}

PATIENT POPULATION

Thirty seven consecutive patients with one vessel coronary artery disease ( $\geqslant 50 \%$ diameter stenosis on visual analysis of the angiogram), suitable for balloon angioplasty, and normal left ventricular function, were studied. Patients were excluded if they had multilesion one vessel disease or total coronary occlusion, prior coronary artery bypass surgery, ECG evidence of left ventricular hypertrophy, or the inability to perform a bicycle exercise test. Patients were included after providing informed consent.

The study protocol was approved by the institutional medical ethics committee of the Academic Medical Center. 
${ }^{99 \mathrm{~m}}$ Tc MIBI MYOCARDIAL TOMOGRAPHY

${ }^{99 \mathrm{~m}}$ Tc MIBI SPECT was performed according to a two day stress/rest protocol, one week before angioplasty. All patients performed a symptom limited exercise test on a calibrated bicycle in the upright position, in a fasting state, at day 1 . Each patient started at a workload of $50 \mathrm{~W}$, increasing by $25 \mathrm{~W}$ every two minutes. Twelve lead ECG, heart rate, and blood pressure were obtained at a two minute interval. At peak exercise, an intravenous bolus of approximately $400 \mathrm{MBq}{ }^{99 \mathrm{~m}} \mathrm{Tc}$ MIBI was given. At day 2, 24-72 hours after the stress test, $400 \mathrm{MBq}^{99 \mathrm{~m}}$ Tc MIBI was injected at rest. Image acquisition was started one to two hours after administration of ${ }^{99 \mathrm{~m}} \mathrm{Tc}$ MIBI, with the patient in a prone position. Acquisition was performed using a three headed gamma camera (Multi-SPECT3, Siemens $\mathrm{GmbH}$, Germany), equipped with a high resolution, parallel hole, low energy collimator. All images were stored in a $64 \times 64$ matrix. The acquisition was obtained in a $360^{\circ}$ circular orbit, $120^{\circ}$ per head, according to a step and shoot procedure; 20 frames per head of 45 seconds per frame. Standard filtered back projection was performed without applying attenuation correction. Image reconstruction was performed on the HERMES workstation (Nuclear Diagnostics, Stockholm, Sweden).

Transaxial slices were evaluated by consensus of a panel of three experienced nuclear physicians who were blinded to angiographic and Doppler data. The images were scored for the presence or absence of reversible perfusion defects. Perfusion abnormalities were related to the perfusion beds of the three coronary arteries. ${ }^{99 \mathrm{~m}} \mathrm{Tc}$ MIBI SPECT was performed one week before cardiac catheterisation, three weeks after balloon angioplasty, and at six months' follow up.

\section{CARDIAC CATHETERISATION}

All patients received aspirin $(100 \mathrm{mg}$ ) and lorazepam $(1 \mathrm{mg})$ orally, before cardiac catheterisation. Anti-ischaemic medication (nitrates, $\beta$ blockers, calcium antagonists) and antiplatelet agents were continued until cardiac catheterisation. Cardiac catheterisation was performed in all patients by the percutaneous femoral approach, using 6 French introduction sheaths. An intravenous bolus of heparin (5000 IU) was administered after the insertion of the sheaths. Additional heparin was given if the procedure lasted more than 90 minutes. Coronary angioplasty was performed according to the standard clinical protocol at our institution. Immediately after balloon angioplasty, coronary angiography was repeated in the same views as before angioplasty. Coronary angiography of the dilated coronary artery was performed at six months' follow up, in the same orthogonal projections.

CORONARY FLOW VELOCITY ANALYSIS

Coronary blood flow velocity measurements were performed with a Doppler angioplasty guide wire (Flowire, Cardiometrics, Mountain View, California, USA) as previously described and validated. ${ }^{7}$ The Doppler signals were proc- essed by a real time spectral display, using a fast Fourier transformation (Flowmap, Cardiometrics) and were recorded on super VHS videotape. The location of the Doppler guide wire was documented by cineangiography.

The tip of the Doppler guide wire was placed distal to the stenosis and manipulated until a reliable and traceable flow velocity profile was obtained. Blood flow velocities, using average peak velocity, were recorded at baseline and during hyperaemia. Hyperaemia was induced by intracoronary administration of a bolus of adenosine $(18 \mu \mathrm{g}$ for the left coronary artery and $12 \mu \mathrm{g}$ for the right coronary artery). CFVR was calculated as the ratio of hyperaemic to baseline average peak flow velocity. After angioplasty, CFVR was assessed proximally in a visually non-stenotic reference coronary artery. The rCFVR was expressed as the ratio between the dCFVR and the CFVR measured in the reference coronary artery. The rCFVR before balloon angioplasty was calculated using the CFVR of the reference coronary artery, obtained immediately after balloon angioplasty. dCFVR and rCFVR were assessed before angioplasty, immediately after angioplasty, and at six months' follow up at the same site as before angioplasty.

QUANTITATIVE CORONARY ANGIOGRAPHY

Offline analysis was performed using an automated contour detection algorithm (Artrek system, ADAC Laboratories, Milpitas, California, USA). The outer diameter of the fluid filled guiding catheter, filmed in the centre of the view, was used as a scaling device. Minimal lumen diameter $(\mathrm{mm})$ and per cent diameter stenosis were calculated using two orthogonal projections of the coronary lesion during the end diastolic phase. Per cent diameter stenosis was defined as the ratio between minimal lumen diameter and the mean diameter of the normal proximal and distal reference segments. A lesion was considered intermediate in the range of $30-75 \%$ diameter stenosis. Restenosis was defined as $>50 \%$ diameter stenosis.

\section{STATISTICAL METHODS}

All results are expressed as mean (SD), unless otherwise indicated. A two tailed $t$ test was used to evaluate differences between geometric dimensions and blood flow velocity parameters before balloon angioplasty, directly after balloon angioplasty, and at six months' follow up (late follow up). The areas under the curve of the receiver operating characteristic curves were used to determine best cut off values for the angiographic variables and the intracoronary Doppler derived parameters in relation to the results of the myocardial perfusion imaging. The agreement between Doppler derived indices and the results of perfusion scintigraphy was calculated by the sum of the true positives and the true negatives divided by the total number of observations. Forward stepwise logistic regression was used to predict the result of perfusion scintigraphy from angiographic and blood flow velocity parameters. Statistical correlation between continuous data 
Table 1 Baseline characteristics of patients

\begin{tabular}{lll}
\hline & $\begin{array}{l}\text { Number of patients } \\
(n=37)\end{array}$ & $\%$ \\
\hline $\begin{array}{l}\text { Age (years) (mean (SD)) } \\
\text { (range) }\end{array}$ & $57(9)(37-70)$ & \\
Male/female & $31 / 6$ & $84 / 16$ \\
Previous MI & 12 & 32 \\
Functional class (CCS) & & \\
I & 9 & 24 \\
II & 9 & 24 \\
III & 18 & 49 \\
IV & 1 & 3 \\
Diabetes & 0 & 0 \\
Smoking & 21 & 57 \\
Hypertension & 9 & 24 \\
Total cholesterol > 6.5 mmol/1 & 18 & 49 \\
Medication & & \\
$\quad \beta$ blockers & 29 & 78 \\
Calcium antagonists & 30 & 81 \\
Nitrates & 27 & 73 \\
Coronary artery involved & & \\
LAD & 12 & 32 \\
LCx & 16 & 43 \\
RCA & 9 & 24 \\
\hline
\end{tabular}

CCS, Canadian Cardiovascular Society; LAD, left anterior descending coronary artery; LCx, left circumflex coronary artery; RCA, right coronary artery.

was calculated by the $\mathrm{Z}$ test. The software used for statistical analysis was Statview for Macintosh (Abacus Concepts, Berkeley, California, USA) version 4.5, and SPSS for Macintosh, version $6.1 .1 ; \mathrm{p}<0.05$ was considered significant.

\section{Results}

Clinical and baseline characteristics of the 37 patients studied are shown in table 1 . A total of $107{ }^{99 \mathrm{~m}}$ Tc MIBI SPECTs were performed (37 patients before angioplasty, 36 patients at three weeks' follow up, and 34 patients at late follow up). At late follow up, two patients had withdrawn their informed consent. dCFVR was measured in all patients before and immediately after balloon angioplasty. At late follow up dCFVR was measured in 33 patients. CFVR of the reference coronary artery was assessed immediately after balloon angioplasty (32 patients) and at late follow up (27 patients).

Table 2 Mean (SD) coronary blood flow velocity parameters and arterial dimensions before, immediately after balloon angioplasty, and at six months'follow up

\begin{tabular}{|c|c|c|c|c|}
\hline \multirow[b]{2}{*}{ Dilated coronary artery } & \multirow[t]{2}{*}{ Before angioplasty } & After angioplasty & \multicolumn{2}{|c|}{ Follow up } \\
\hline & & & & \\
\hline$\%$ diameter stenosis & $77 \quad(11)^{\star}$ & $29 \quad(13) \dagger$ & 37 & $(22) \ddagger$ \\
\hline Minimal lumen diameter $(\mathrm{mm})$ & $0.69(0.34)^{\star}$ & $1.98(0.44) \dagger$ & 1.6 & $(0.65) \ddagger$ \\
\hline Baseline APV & $12.8(5.3)^{\star}$ & $17.3(11.1)$ & 19.6 & $(11.2) \ddagger$ \\
\hline Hyperaemic APV & $19.9(12.2)^{\star}$ & $46.3(19.3)$ & 60.8 & $(31.4) \ddagger$ \\
\hline dCFVR & $1.4(0.6)^{\star}$ & $2.9(1.0)$ & 3.2 & $(0.9) \ddagger$ \\
\hline \multicolumn{5}{|l|}{ Normal reference coronary artery } \\
\hline Baseline APV & - & $19.6(11.2)$ & 18.0 & $(7.6)$ \\
\hline Hyperaemic APV & - & $61.0 \quad(31.1)$ & 57.1 & $(20.3)$ \\
\hline CFVR & - & $3.2(0.5)$ & 3.3 & $(0.7)$ \\
\hline Relative coronary flow velocity reserve & $0.49(0.20)^{\star}$ & $0.98(0.29)$ & 0.90 & $(0.24) \ddagger$ \\
\hline
\end{tabular}

${ }^{\star} \mathrm{p}<0.05$, compared to paired values directly after balloon angioplasty; $\mathrm{tp}<0.05$, compared to paired values at late follow up; $\neq \mathrm{p}<0.05$, compared to paired values before balloon angioplasty. $\mathrm{APV}$, average peak flow velocity $(\mathrm{cm} / \mathrm{s})$.

Table 3 Angiographic and Doppler derived indices for intermediate lesions and the total range of coronary artery stenosis

\begin{tabular}{|c|c|c|}
\hline & Total & Intermediate lesions \\
\hline$\%$ diameter stenosis & $48 \quad(28)(0-98)$ & (16) $(30-75)$ \\
\hline Minimal lumen diameter $(\mathrm{mm})$ & $1.41(0.78)(0.14-2.92)$ & $1.38(0.54)(0.61-2.62)$ \\
\hline dCFVR & $2.3(1.0)(0.9-5.1)$ & $2.3(0.8)(0.9-3.7)$ \\
\hline rCFVR & $0.78(0.33)(0.23-1.70)$ & $0.75(0.26)(0.26-1.21)$ \\
\hline
\end{tabular}

Values are mean (SD) (range).
ANGIOGRAPHIC AND CORONARY FLOW VELOCITY DATA

Coronary flow velocity and angiographic data before, immediately after balloon angioplasty, and at late follow up are summarised in table 2 . The dCFVR improved immediately after balloon angioplasty to values that were in the range of the CFVR measured in the reference coronary artery. At late follow up there were seven patients with a restenosis. No significant differences were found when comparing the dCFVR immediately after balloon angioplasty with dCFVR at late follow up.

Coronary flow velocity and angiographic parameters for the total range of lesion severity and the intermediate lesions of the pooled data (pre-angioplasty, immediately after angioplasty, and at late follow up) are provided in table 3 .

RELATION BETWEEN ANGIOGRAPHIC AND BLOOD FLOW VELOCITY PARAMETERS

There was a strong linear relation between dCFVR and rCFVR in the total range of coronary artery severity $\quad(r=0.93$, $\mathrm{p}<0.0001)$. For lesions of intermediate severity, there was also a strong linear relation between dCFVR and rCFVR $(r=0.87$, $\mathrm{p}<0.001)$. The dCFVR showed a good correlation with per cent diameter stenosis $(r=-0.73, \mathrm{p}<0.0001)$ and minimal lumen diameter $(r=0.72, \mathrm{p}<0.0001)$ in the total range of coronary narrowing. The overall correlations using rCFVR were equal to the overall correlations using dCFVR (per cent diameter stenosis $r=-0.73, \mathrm{p}<0.0001$; minimal lumen diameter $r=0.73, \mathrm{p}<0.0001$ ).

ANGIOGRAPHIC AND DOPPLER DERIVED ESTIMATES OF CORONARY LESION SEVERITY IN RELATION TO ${ }^{99 \mathrm{~m}}$ TC MIBI SPECT

Before angioplasty, reversible defects in the vascular bed of the coronary arteries studied were present in 29/37 patients (78\%), after balloon angioplasty in $6 / 36$ patients (17\%), and at late follow up in 9/34 patients (26\%). Six out of the seven patients with a restenosis at late follow up had a reversible defect.

The areas under the curves and the best cut off values of the calculated receiver operating curves of per cent diameter stenosis, minimal lumen diameter, dCFVR, and rCFVR are shown in table 4 . There were no significant differences between the areas of the receiver operating characteristic curves of these variables both in intermediate lesions as well as in the total range of coronary artery stenosis.

AGREEMENT BETWEEN ${ }^{99 \mathrm{~m}}$ TC MIBI SPECT AND OTHER PARAMETERS

The agreement between dCFVR and the presence or absence of reversible defects in the total range of per cent diameter stenosis at a threshold of 1.9 before, immediately after balloon angioplasty, and at late follow up was $78 \%$, $78 \%$, and $81 \%$, respectively. The overall agreement was $81 \%$ (table 4 ). The agreement between rCFVR and reversible perfusion defects in the total range of coronary narrowings at a threshold of 0.65 before, after balloon 
Table 4 Prediction of ${ }^{99 m}$ Tc MIBI SPECT results

\begin{tabular}{|c|c|c|c|c|c|}
\hline & $A U C$ & $B C V$ & $\begin{array}{l}\text { Sensitivity } \\
(\%)\end{array}$ & $\begin{array}{l}\text { Specificity } \\
(\%)\end{array}$ & $\begin{array}{l}\text { Agreement } \\
(\%)\end{array}$ \\
\hline \multicolumn{6}{|c|}{ Total range of $\%$ diameter stenosis $0-98 \%$} \\
\hline$\%$ diameter stenosis & $0.78(0.05)$ & 50 & 66 & 80 & 82 \\
\hline Minimal lumen diameter $(\mathrm{mm})$ & $0.75(0.05)$ & 1.3 & 70 & 80 & 81 \\
\hline dCFVR & $0.85(0.04)$ & 1.9 & 67 & 86 & 81 \\
\hline rCFVR & $0.85(0.04)$ & 0.65 & 78 & 89 & 85 \\
\hline \multicolumn{6}{|c|}{ Intermediate range of $\%$ diameter stenosis $30-75 \%$} \\
\hline$\%$ diameter stenosis & $0.79(0.07)$ & 45 & 75 & 78 & 78 \\
\hline Minimal lumen diameter ( $\mathrm{mm})$ & $0.71(0.08)$ & 1.3 & 72 & 71 & 72 \\
\hline dCFVR & $0.72(0.08)$ & 2.0 & 62 & 82 & 73 \\
\hline rCFVR & $0.77(0.07)$ & 0.65 & 70 & 85 & 78 \\
\hline
\end{tabular}

AUC, area under the receiver operating curve; BCV, best cut off value.

Table 5 Independent explanatory variables for the scintigraphic results

\begin{tabular}{lrrr}
\hline & Coefficient $b$ & $\begin{array}{l}\text { Standard } \\
\text { error }(b)\end{array}$ & p Value \\
\hline Total range of coronary artery stenosis & & \\
$\quad$ Constant & -2.0246 & 0.4156 & \\
\% diameter stenosis & 1.7667 & 0.6945 & 0.0101 \\
$\quad$ rCFVR & 2.0757 & 0.7086 & 0.0034 \\
Intermediate lesion severity & & & \\
$\quad$ Constant & -1.0498 & 0.4392 & \\
rCFVR & 2.0614 & 0.7306 & 0.0048 \\
\hline
\end{tabular}

angioplasty, and at late follow up was $81 \%$, $81 \%$, and $93 \%$, respectively, with an overall agreement of $85 \%$. The overall agreement for lesions in the right coronary artery was lower than the agreement for lesions in the left anterior descending coronary artery or in the left circumflex coronary artery $(68 \% v 89 \%$ and $81 \%$, respectively, $\mathrm{p}<0.005)$. For lesions of intermediate severity, the agreement between $\mathrm{dCFVR}$ and myocardial perfusion imaging was $73 \%$ and increased to $78 \%$ using rCFVR. The overall agreement in the total range of coronary artery stenosis did not differ in patients with (all non-Q wave, included more than one year after myocardial infarction) or without myocardial infarction using dCFVR $(79 \% v 82 \%)$ or rCFVR ( $86 \%$ v 84\%).

\section{REGRESSION ANALYSIS}

Per cent diameter stenosis, minimal lumen diameter, dCFVR, and rCFVR were transformed to dichotomous variables (positive or negative) using the best cut off values of the receiver operating characteristic curves. Forward stepwise regression analysis was used to calculate the model which best described the outcome of the scintigraphic test, using the above mentioned parameters as explanatory variables. The results of the forward logistic stepwise regression analysis are shown in table 5 . For the total range of coronary artery stenosis both per cent diameter stenosis and rCFVR were independent predictors for the outcome of the scintigraphic results. In intermediate lesions, rCFVR was the only independent explanatory variable for the results of myocardial perfusion scintigraphy.

\section{Discussion}

Our study shows that there is a good agreement between intracoronary Doppler derived coronary flow reserve and the results of myocardial perfusion scintigraphy, in a selected cohort of patients with one vessel disease and a normal left ventricular function. Furthermore, rCFVR and per cent diameter stenosis are independent predictors of myocardial perfusion scintigraphy, in this category of patients.

dCFVR AND MYOCARDIAL PERFUSION SCINTIGRAPHY

Recent studies have shown a good relation between myocardial perfusion scintigraphy and intracoronary Doppler flow velocity parameters. ${ }^{8-1115}$ However, the results are not uniform regarding both the best cut off values for the distal coronary flow reserve as well as the agreement between the invasive intracoronary diagnostic technique and non-invasive myocardial perfusion scintigraphy. Miller and colleagues found an overall agreement of $89 \%$ between pharmacologic (adenosine and dipyridamole) induced stress ${ }^{99 \mathrm{~m}}$ Tc MIBI SPECT and dCFVR (cut off value 2.0) in a cohort of patients with single and multivessel disease. ${ }^{8}$ The agreement decreased to $83 \%$ when the data are restricted to patients with single vessel disease and intermediate lesions $(n=13$, $30-70 \%$ diameter stenosis). Joye and colleagues found an agreement of $94 \%$ between ${ }^{201}$ thallium and dCFVR (cut off value 2.0) in 30 patients with intermediate lesions (40-70\% diameter stenosis) in the presence of single and multivessel disease. ${ }^{9}$ Stress ${ }^{201}$ thallium was performed using a physical exercise test as well as during hyperaemia induced by dipyridamole. Deychak and colleagues reported an agreement of $96 \%$ between ${ }^{201}$ thallium and dCFVR in 17 patients with a positive ${ }^{201}$ thallium result. ${ }^{11}$ dCFVR (cut off value 1.8) was measured in 16 coronary arteries with a stenosis (55-85\% diameter stenosis) and a corresponding reversible perfusion defect, and in 11 coronary arteries with no perfusion defect. This agreement, which is almost perfect, may be because only patients with a reversible defect and an abnormal angiogram were included. Tron and colleagues found an agreement of $84 \%$ between stress (pharmacologic and physical exercise) and myocardial perfusion imaging ( ${ }^{201}$ thallium and ${ }^{99 \mathrm{~m}} \mathrm{Tc}$ MIBI SPECT) and dCFVR in a patient cohort with single and multivessel disease. ${ }^{10}$ Heller and colleagues reported an agreement of $84 \%$ between dCFVR (cut off value 1.7) and ${ }^{201}$ thallium imaging in patients with single and multivessel disease, in a multicentre study. ${ }^{15}$

Compared to the studies mentioned above, the agreement in our study between Doppler derived indices and myocardial perfusion scintigraphy both in intermediate lesions as well as 
in the total range of coronary narrowings was less. The present study focused on patients with single vessel disease, who underwent a uniform stress protocol. Moreover, the data from our patients included the results obtained pre-angioplasty, immediately after balloon angioplasty, and late follow up. In particular, CFVR immediately after angioplasty may be temporarily impaired because of a reduction of hyperaemic flow velocity (microembolisation, vasoconstriction) or increased baseline flow velocity (disturbed autoregulation), while perfusion scintigraphy was performed three weeks after the procedure. ${ }^{16-18}$ The inclusion of postintervention data may have contributed to the observed differences between previous studies and our investigation.

It can be anticipated that the agreement between CFVR and perfusion scintigraphy will be lower in patients with multivessel disease. Both myocardial perfusion scintigraphy and rCFVR are based on differences between the vascular territories. Therefore, there are inherent diagnostic limitations of myocardial perfusion scintigraphy and rCFVR in multivessel disease. In this respect a selective intracoronary technique using either distal pressure or flow is potentially advantageous for the evaluation of coronary lesion severity.

RELATIVE CORONARY FLOW RESERVE AND

MYOCARDIAL PERFUSION SCINTIGRAPHY

A $50 \%$ decrease in regional flow during hyperaemia is associated with the appearance of ${ }^{201}$ thallium defects, in a canine model. ${ }^{19}$ It is conceivable to expect similar findings for ${ }^{99 \mathrm{~m}} \mathrm{Tc}$ MIBI as the distribution of ${ }^{99 \mathrm{~m}}$ Tc MIBI is also proportional to the myocardial blood flow. ${ }^{20}$ In our study, rCFVR $<0.65$, a poststenotic flow reduction of $35 \%$ or more, is associated with the presence of ischaemia on ${ }^{99 \mathrm{~m}} \mathrm{Tc}$ MIBI SPECT.

Heller and colleagues introduced the flow ratio index in their recent study on the relation between intracoronary Doppler derived indices and stress perfusion imaging. ${ }^{15}$ This inverse equivalent of rCFVR is calculated as the ratio between the coronary flow velocity in a normal reference vessel and the distal coronary flow velocity of the diseased coronary artery. The rCFVR calculated from their data gives a best cut off value of 0.59 ( $41 \%$ flow reduction) for the presence of ischaemia on the ${ }^{201}$ thallium scan and is similar to our results. However, rCFVR gives a direct measurement of the regional decreased blood flow; rCFVR of 0.7 means a flow reduction of $30 \%$ but a flow ratio index of 1.4 .

rCFVR and the per cent diameter stenosis are independent predictors of the results of the myocardial perfusion scintigraphy in the total range of coronary artery stenosis. Moreover, in this study, rCFVR is the only independent explanatory variable for the result of the myocardial perfusion scintigraphy, in intermediate lesions (table 5). This emphasises the complementary role of measuring both parameters in an individual patient.

Experimental and clinical data have suggested that rCFVR is suitable for haemody- namic evaluation of coronary lesions as it is relatively independent of haemodynamic variables. $^{621}$ We found that rCFVR is not a better predictor for the results of myocardial perfusion scintigraphy compared to dCFVR. This finding may be related to the selected patient cohort - that is, patients with single vessel coronary artery disease and a normal left ventricular function. At present there are no data concerning the diagnostic value of the rCFVR in patients with a compromised haemodynamic cardiac condition.

\section{CLINICAL IMPLICATIONS}

Our study shows a good overall agreement between Doppler derived estimates of coronary lesion severity and perfusion scintigraphy. This indicates that intracoronary Doppler derived indices may serve as an alternative for diagnostic purposes. This is important in view of the increasing number of angioplasties that are performed as a one stage procedure following the diagnostic angiography. ${ }^{22}$ Moreover, recent studies indicated that in patients with normal translesional haemodynamic data it is safe to postpone angioplasty. ${ }^{23}$ Moreover, the one year cardiac event rate in patients with a normal or equivocal ${ }^{99 \mathrm{~m}} \mathrm{Tc}$ MIBI SPECT is low. ${ }^{24}$

Nevertheless, discordant results were present between ${ }^{99 \mathrm{~m}} \mathrm{Tc}$ MIBI SPECT and intracoronary Doppler flow indices in approximately $20 \%$ of the patients. At present, the interpretation of these discordant results for patient management are unknown and need to be evaluated in a prospective trial.

\section{LIMITATIONS}

The patients' medication was not uniform in our study. This may have contributed to the observed differences with other studies. Intracoronary blood flow velocity reserve analysis is a sensitive technique for the detection of changes in blood flow. However, this technique is prone to technical failure and is influenced by changes in heart rate, contractility, preload, and afterload. ${ }^{12} 1425$

The rCFVR before balloon angioplasty was calculated using the reference coronary flow velocity assessed immediately after balloon angioplasty. As reported by Foley and colleagues in patients with stable angina, successful angioplasty is not associated with any alterations in the CFVR of angiographically normal non-dilated coronary arteries. ${ }^{26}$ The reference coronary flow velocity was assessed in a visually normal coronary artery. However, this does not exclude diffuse or microvascular coronary artery disease.

Perfusion scintigraphy was performed at three weeks to minimise a higher false positive rate caused by the effect of hibernating myocardium. ${ }^{27}$ If perfusion scintigraphy is performed within 24 hours after balloon angioplasty the agreement between already "normalised" CFVR and the results of the perfusion scintigraphy would be underestimated. However, it is uncertain if the angiographic and Doppler derived indices immediately after angioplasty represent the cardiac condition at three weeks' follow up. Nevertheless, in our 
study dCFVR improved immediately after successful balloon angioplasty compared to preballoon angioplasty. Additionally, it was not significantly different at late follow up.

Regional hyperaemia induced by an intracoronary bolus of adenosine may not be comparable to hyperaemia induced by bicycle exercise. This may have attributed to the observed differences between ${ }^{99 \mathrm{~m}}$ Tc MIBI SPECT and Doppler derived indices.

Our study relates to patients with single vessel disease in a single centre. This limits extrapolation of the results to other patient cohorts and requires confirmation by other investigators.

CONCLUSIONS

There is a good agreement between intracoronary Doppler derived indices of lesion severity and ${ }^{99 m}$ Tc MIBI SPECT in patients with single vessel disease and normal left ventricular function. Moreover, rCFVR and per cent diameter stenosis are independent predictors of ${ }^{99 \mathrm{~m}} \mathrm{Tc}$ MIBI SPECT. Consequently, CFVR provides independent information of coronary lesion severity at the time of angiography. In a majority of the patients with intermediate coronary artery stenosis, intracoronary Doppler derived indices could obviate the need for additional myocardial perfusion imaging. However, the discordant results in approximately $20 \%$ of the patients need to be evaluated in a prospective trial.

The authors would like to thank the technical and nursing staff of the cardiac catheterisation laboratory (M Meesterman, head) and the department of nuclear medicine (A Lagerwaard, head) and the department of nuclear medicine (A Lagerwaard, head)
for their enthusiastic and skilled assistance. JJP is a clinical investigator for the Netherlands Heart Foundation (grant investigator for the
number D96.020).

1 White CW, Wright CB, Doty DB, et al. Does visual interpretation of the coronary arteriogram predict the
physiologic importance of a coronary stenosis? $N$ Engl $\mathcal{F}$ physiologic importance

2 Zir LM, Miller SW, Dinsmore RE, et al. Interobserver variability in coronary angiography. Circulation 1976;53:62732.

3 Marcus ML, Skorton DJ, Johnson MR, et al. Visual estimates of percent diameter coronary stenosis: "a battered gold standard". 7 Am Coll Cardiol 1988;11:882-5.

4 Zijlstra F, Fioretti P, Reiber JHC, et al. Which cineangiographically assessed anatomic variable correlates best with functional measurements of stenosis severity? A comparison of quantitative analysis of the coronary cineangiogram with measured coronary flow reserve and exercise/ redistribution thallium-201 scintigraphy. $₹ \mathrm{Am}$ Coll Cardiol 1988;12:686-91.

5 Topol EJ, Ellis SG, Cosgrove DM, et al. Analysis of coronary angioplasty practice in the United States with an insurance-claims data base. Circulation 1993;87:1489-97.
6 Gould KL, Kirkeeide RL, Buchi M. Coronary flow reserve as a physiologic measure of stenosis severity. $\mathcal{F}$ Am Coll Cardiol 1990;15:459-74

7 Doucette JW, Corl D, Payne HM, et al. Validation of a Doppler guide wire for intravascular measurement of coronary artery flow velocity. Circulation 1992;85:1899-911.

8 Miller DD, Donohue TJ, Younis LT, et al. Correlation of pharmacological $99 \mathrm{mTc}$-sestamibi myocardial perfusion imaging with poststenotic coronary flow reserve in patients with angiographically intermediate coronary artery stenwith angiographically intermediate

9 Joye JD, Schulman DS, Lasorda D, et al. Intracoronary Doppler guide wire versus stress single-photon emission computed tomographic thallium-201 imaging in assessment of intermediate coronary stenoses. $\mathcal{F}$ Am Coll Cardiol 1994;24:940-7.

10 Tron C, Donohue TJ, Bach RG, et al. Comparison of pressure-derived fractional flow reserve with poststenotic pressure-derived fractional flow reserve with poststenotic cardial perfusion imaging results. Am Heart f 1995;130: cardial perf

11 Deychak YA, Segal J, Reiner JS, et al. Doppler guide wire flow-velocity indexes measured distal to coronary stenoses associated with reversible thallium perfusion defects. Am Heart f 1995;129:219-27.

12 McGinn AL, White CW, Wilson RF. Interstudy variability of coronary flow reserve. Circulation 1990;81:1319-30.

13 Hoffman JIE. A critical view of coronary reserve. Circulation 1987;75(suppl I):I-6-11.

14 Klocke FJ. Measurements of coronary flow reserve: defining pathophysiology versus making decisions about patient care. Circulation 1987;76:1183-9.

15 Heller LI, Cates C, Popma J, et al. Intracoronary Doppler assessment of moderate coronary artery disease. Circulation 1997;96:484-90.

16 Uren NG, Crake T, Lefroy DC, et al. Delayed recovery of coronary resistive vessel function after coronary angioplasty. F Am Coll Cardiol 1993;21:612-21.

17 van Liebergen RAM, Piek JJ, Koch KT, et al. Immediate and long-term effect of balloon angioplasty or stent implantation on the absolute and relative coronary blood flow velocity reserve. Circulation 1998;98:2133-40.

18 Wilson RF, Johnson MR, Marcus ML, et al. The effect of coronary angioplasty on coronary flow reserve. Circulation 1988;77:873-85.

19 Gould KL. Noninvasive assessment of coronary stenoses by myocardial perfusion imaging during pharmacologic coronary vasodilatation. Am $\mathcal{f}$ Cardiol 1978;41:267-78.

20 Okada RD, Glover D, Gaffney T, et al. Myocardial kinetics of technetium-99m-hexakis-2-methoxy-2-methylpropylisonitrile. Circulation 1988;78:491-8.

21 Baumgart D, Haude M, Goerge G, et al. Improved assessment of coronary stenosis severity using the relative flow velocity reserve. Circulation 1998;98:40-6.

22 Rozeman Y, Gilon D, Zelingher J, et al. One-stage coronary angiography and angioplasty. Am 7 Cardiol 1995;75:30-3.

23 Kern MJ, Donohue TJ, Aguirre FV, et al. Clinical outcome of deferring angioplasty in patients with normal translesional pressure-flow velocity measurements. $\mathcal{F} \mathrm{Am}$ Coll Cardiol 1995;25:178-87.

24 Berman DS, Hachamovitch R, Kiat $\mathrm{H}$, et al. Incremental value of prognostic testing in patients with known or suspected ischemic heart disease: a basis for optimal utilization of exercise technetium-99m ses dial perfusion single-photon emission computed tomography. F Am Coll Cardiol 1995;26:639-47.

25 Hoffman JIE. Maximal coronary flow and the concept of coronary vascular reserve. Circulation 1984;70:153-9.

26 Foley JB, Watson KR, Chisholm RJ. Impact of coronary angioplasty on coronary vasodilator response of normal nondilated coronary arteries in patients with stable angina. Am 7 Cardiol 1997;75:1070-1.

27 Miller DD, Verani MS. Current status of myocardial perfusion imaging after percutaneous transluminal coronary angioplasty. F Am Coll Cardiol 1994;24:260-6. 